\title{
ENHANCED SYSTEM FOR COMPUTER-AIDED DETECTION OF MRI BRAIN TUMORS
}

\author{
${ }^{1}$ Dr. Umar Alqasmi, Ammar Alzuhair², Abduallah Bama'bad ${ }^{3}$ \\ ${ }^{1}$ Biomedical Engineering at King Abdul-Aziz University, KSA \\ ${ }^{2,3}$ Electrical and Biomedical Engineering in King Abul-Aziz University, KSA
}

\begin{abstract}
The brain images are indicating what condition the brain has. The objective of this research is to design a software that will automatically classifies the brain images to their associated disorders. In order to achieve the objective of this research, a database for training and testing the software of brain images must to be found. In this research we have 105 number of images in data set. In order to differentiate between the classes of those brain images, features had to be extracted from the images. Then, images will be classified into two classes normal and abnormal by using SVM and KNN classifier. The features that were extracted were used in the classification process. The classifiers performed really well, whereas the SVM classifier performed better since its accuracy is $100 \%$ on testing set. In the end, the software was successful in separating the two classes.
\end{abstract}

\section{KEYWORDS}

Computer-Aided Detecting, MRI, Brain Tumor, MATLAB

\section{INTRODUCTION}

Brain can suffer from a number of diseases or conditions such as Epilepsy, Cancer, and Strokes. Medical imaging is one of the best ways to determine the kind of the disease, its location, and severity. Another method of testing brain functionality is EEG "electroencephalogram" recordings. In this paper, a database of brain images is obtained to be worked on. The data consists of brain images of patients with brain-tumor and patient without brain-tumor. For example, glioblastoma is one of the diseases introduced in the database brain images. Glioblastoma, also known as glioblastoma multiforme (GBM), is the most aggressive cancer that begins within the brain [1]. Initially, signs and symptoms of glioblastoma are nonspecific [1]. They may include headache, personality changes, nausea, and symptoms similar to those of a stroke [1].

The number of brain images presented to a neurologist and the number of brain conditions makes it difficult to instantly diagnose the patient. Brain can encounter several conditions that alter the shape of the brain, which is seen from the images taken by either Magnetic Resonance Image (MRI) or X-rays. Training a software to know all brain conditions will help physicians diagnose the patients' conditions faster which will increase the survival rate of the patients when the appropriate treatment is administered. It can also serve as a secondary screening method to help locate tumors for possible biopsy. The Objective of this research is to design a computer diagnosis support software for neurologists. Another objective of this research is to use statistical spatial and frequency domain features to study possible significant features for this particular type of disease. The main problem to be solved in this research, is to design a fast software that can detect tumors from normal brain tissue. 
Signal \& Image Processing: An International Journal (SIPIJ) Vol.11, No.1, February 2020

\section{Literature REVIEW}

SAINI, Pankaj Kr et al [2] discusses the process of detecting tumor and cancer cells from MRI images of the brain using mathematical morphology. They have suggested an effective classification system based on density-based clustering approach to detect the brain tumor. The MATLAB simulation is carried on different brain images and tumor is detected using OTSU'S method for image segmentation and optimal global thresholding [2].

ABD-ELLAH, Mahmoud Khaled, et al [3] discussed the presence and absence of the brain tumor in the first stage, and then classified it into a benign or malignant tumor using the Computer Aided-Detection (CAD) system. They evaluated the CAD system in terms of accuracy for both detection and classification, it achieved an accuracy rate of $100 \%$ in the classification [3].

RAMARAJU, P. V, et al [4] discussed the use of an automatic method to classify the stage of the tumor in Magnetic Resonance Images (MRI) into normal or benign or malignant tumors using Probabilistic neural network and Graphical user interface (GUI). The MATLAB program was used to detect and extract the tumor from MRI images. Also, they calculated parameters like area, mean, standard deviation and entropy [4].

MOHSEN, Heba; EL-DAHSHAN, et al [5] discussed the application of hybrid machine learning technology on 141 magnetic resonance (MRI) images of the brain using the CAD system in order to detect the presence of tumors in the brain and categorize them into natural and abnormal (benign or malignant), where the accuracy rate was $99 \%$ for both training and testing images in In this technique. Several computational methods were used in this technique, including: the feedback pulse-coupled neural network for image segmentation, the discrete wavelet transform for features extraction, the principal component analysis for reducing the dimensionality of the wavelet coefficients, and the feed forward backpropagation neural network to classify inputs into normal or abnormal [5].

R. Ramteke, et al. discusses the classification of brain images using K-nearest neighbor [6]. Like any other research, their methodology consists mainly of finding a database, feature extraction and classification. The features they used depend on texture [6]. They used occurrence matrix and Haralick features that characterizes the color and texture of an image [6]. Haralick features that are used in this paper include contrast, homogeneity, correlation, entropy, and local homogeneity. After feature extraction, their classification process starts. For their classification, they used KNN classifier which works by classifying each pixel by looking at the class of its nearest neighbor. Their results indicate that their classifier performed well by getting an accuracy of $80 \%$ [6].

S. Chaplot, L. Patnaik, et al [7] discusses the classification of MRI brain images. The researchers used wavelet-based features which decompose data into frequency components [7]. Also, they used DWT in two dimensions. In addition, they used Self-organizing maps (SOM) which is powerful neural network method. SOM is an unsupervised method which means that it takes a series of input patterns, and it learns how to group those patterns by itself. Then, SVM is used in the classification phase. The output of the classifier is either normal image or abnormal image. They actually used a number of (Support Vector Machine) SVM classifiers such as linear SVM classifier and non-linear SVM classifier. Their SVM classifiers resulted in $96.16 \%$ accuracy. [7]This paper shows the different types of SVM classifiers that can be used in the classification process. Also, it helps in giving ideas about features or methods to group certain images together.

Abdullah, N., Ngah, U., et al, supports the idea of using SVM classifiers in the classification of MRI brain images is a written by N. Abdullah et al [8]. Here, they used LabView software instead of MATLAB [8]. LabView is a graphical language used in industry and universities as an 
Signal \& Image Processing: An International Journal (SIPIJ) Vol.11, No.1, February 2020

instrument and analysis software system [8]. In order to classify their brain images, they relied on wavelet transform such as previously discussed researches. Their wavelet transform resulted in 17689 approximation coefficients for each image. Their classifier resulted in $65 \%$ accuracy which they pointed that it might be due to the presence of noise in the original noise. This noise might have caused the classifier to misclassify the data into wrong classes [8]. This paper is helpful in terms of using SVM classifier and determining its abilities and factors that can affect it. However, their use of only one type of features might be the cause of low accuracy classification. This paper teaches that researches should never rely on one specific type of features.

Madina Hamiane, et al, discusses the possibility of classifying MRI brain images using SVM classifier [9]. Such as other papers, they used DWT decomposition for noise filtering [9]. In addition, they used MATLAB thresholding methods and values to decompose the original images into approximations and detail coefficients. The obtained coefficients were then composed together to reconstruct the denoised image. After that, they started image segmentation which is a method to split an image into a group of non-overlapping areas. Then, feature extraction phase started. Here, they used different methods of extracting features including DWT, GLCM, and Gabor filters. Finally, they started the classification process. They used SVM classifier due to its high performance in the literature [9]. Their classifier performed really well, they got an accuracy of $100 \%$ as well as sensitivity and specificity. Their classification was for two classes, normal images and abnormal images.

M.C. Hingane, et al, discusses the possibility to classify brain images using SVM classifiers [10]. Their software consists of two major parts, feature extraction and classification [10]. Their features are based on the GLCM (Grey Level Co-occurrence Matrix) which considers the spatial relationship of pixels. After generating their features, they such as other researches performed a feature selection or reduction algorithm (PCA). Their final step is classification, here they used SVM. SVM is commonly used since it has high accuracy and since it deals with high-dimensional data. Their methodology's structure is similar to other researches in the same field. Based on their conclusion and discussion, their classifier has high accuracy combined with low computation time due to the use of feature reduction function [10].

This research is relevant to ours since here SVM classifier is going to be used. However, their features are different. This research did not specify the actual accuracy of the classifier which is not helpful for a lot of researchers.

Table 1. Summary of previous researches results

\begin{tabular}{|l|l|l|l|}
\hline Reference No. & Used features & Used classifier & Accuracy\% \\
\hline$[6]$ & Haralick features & KNN & 80 \\
\hline$[7]$ & 2D DWT and SOM & SVM & 96.16 \\
\hline$[8]$ & Wavelet Transform & SVM & 65 \\
\hline$[9]$ & $\begin{array}{l}\text { DWT, GLCM, and } \\
\text { Gabor filters }\end{array}$ & SVM & 100 \\
\hline$[10]$ & GLCM & SVM & NAN \\
\hline
\end{tabular}

\section{Methodology}

This paper has a very simple methodology. The process of completing this research consists of finding a brain images database, extracting a few features to differentiate between the classes, and classifying the images based on the extracted features. 
Signal \& Image Processing: An International Journal (SIPIJ) Vol.11, No.1, February 2020

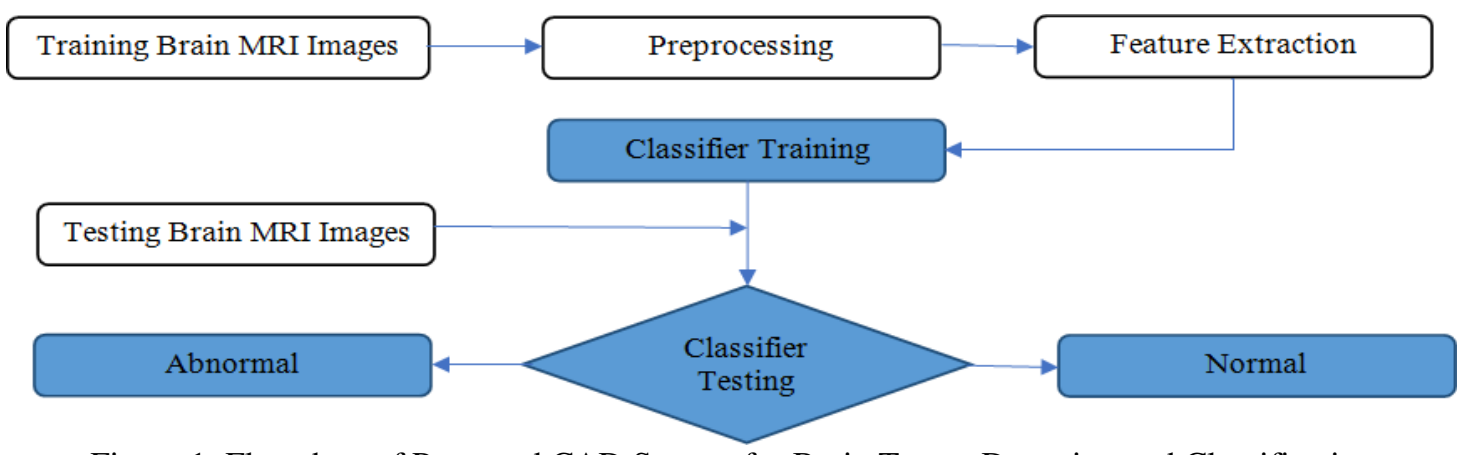

Figure 1: Flowchart of Proposed CAD System for Brain Tumor Detection and Classification

\section{Database Source}

In order to complete this research, a dataset had to be found and used. For this reason, a database of brain images was found from The Cancer Images Archive (TCIA) [1]. It consists of 105 brain images which is excellent for this research's purpose. We used 60 images for learning (30 Normal + 30 Abnormal) and 45 images for testing (30 Normal + 15 Abnormal). It is labeled as well for easy interpretation and use. It has images of two types of the brain one is for normal and the second is for brain tumor Glioblastoma. The Figures 2 show examples of the images in the database.

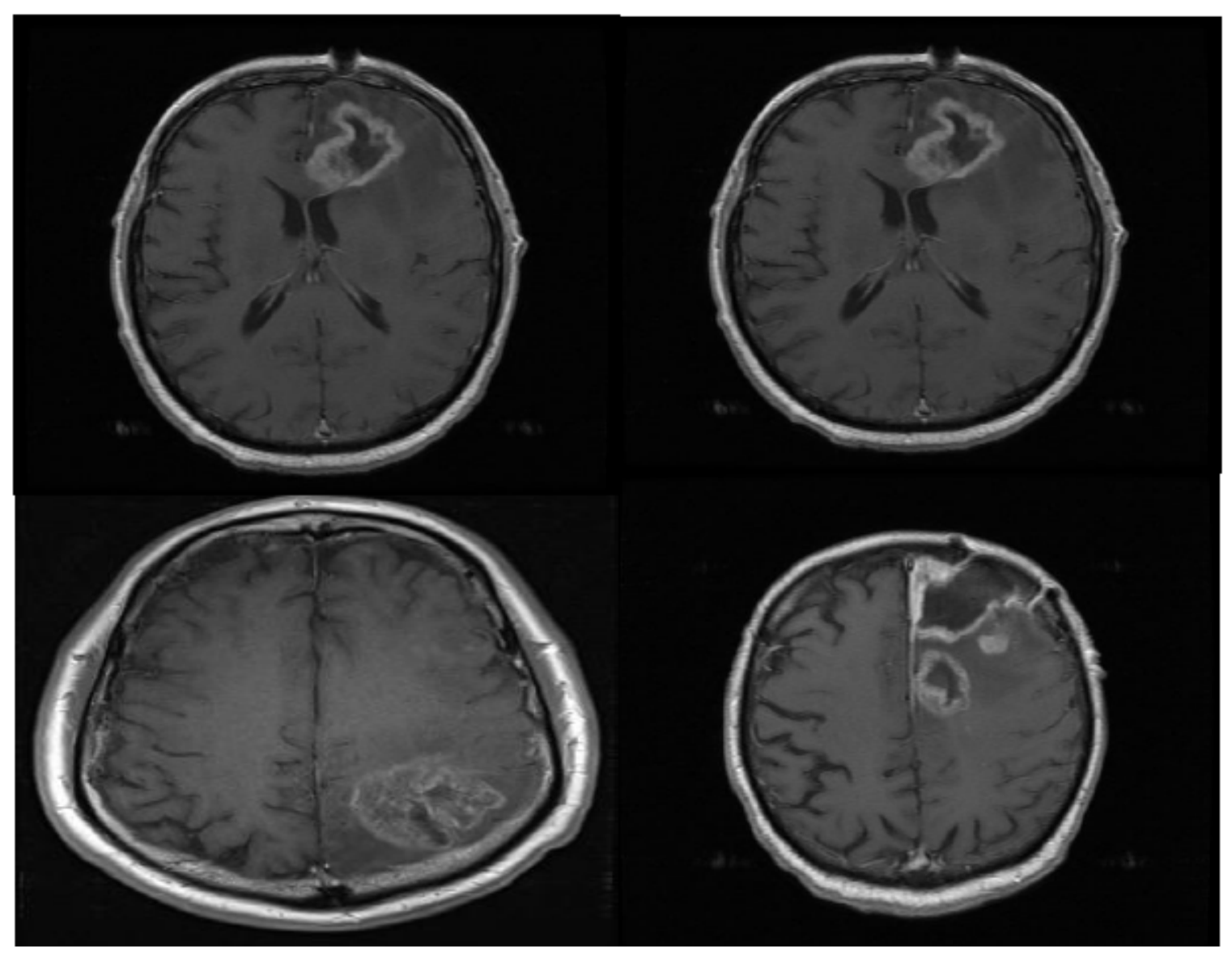

Figure 2: Brain Glioblastoma Images 
Signal \& Image Processing: An International Journal (SIPIJ) Vol.11, No.1, February 2020

The dataset has a good number of images with different angles and views that can be used in the classification and feature extraction process. IT is open source and can be found online. It has full details and description. IT consists of 8798 images that belong to 20 patients [1].

\section{FeATURE EXtraction}

We want to select features which could adjacent between benign and malignant or the features who give us high accuracy, also the features which do not correlate with pathology and features that are not independent. In this research only first order statistical features are considered since they have not been used alone in previous researches. The features that are used are the mean of integration of the actual images, the standard deviation of each image, and the absolute mean of the derivative of each image. In additional, the same features extracted after fast Fourier transform of the images. Features are extracted using MATLAB functions such as "mean", "std", "mode", "median" and "diff" [11]. The mean of the image will determine the most apparent pixel of color and most repeated frequency of an image. The standard deviation will determine how much the other pixels deviate from the mean brightness. Then, the derivative is calculated and used as a feature to know which enhance sudden changes in image brightness. The features that were extracted were plotted in a single figure for each type of tumors. Each feature is obtained from a combination of images that has the same disease but different angles. The combination of images is used in feature extraction that is then used in the classification process.

\section{Classification}

After selecting a database and extracting features from the database's images, it is time for classification of the data. For choosing the classifier that provides best performance, we use two different types of classifiers as follows support vector machine (SVM) with four kernels (Linear, RBF, Polynomial Oreder2 and Order3 and k-voting nearest neighbour (KNN)classifiers [7]. In this step, the data is classified into two classes of brain images into normal and abnormal images. In this paper, about seven classifiers will be used and compared, the best accuracy will be the one used in that software. The classifiers are SVM classifiers that has discriminant function ofthe type "RBF", "POLYNOMIAL", and "LINEAR". The other classifiers that are used are KNN classifiers that have $\mathrm{k}$ values of 1,3 , and 5 [12].

\section{Performance Assessments}

To evaluate classification performance, we calculate ErrorRate, Confusion Matrix, Specificity, Sensitivity, FalseNegative Rate, the Area Under Curve (AUC) from the Receiver operator characteristic (ROC) and overall Accuracy [13]. We focus on the accuracy to make a comparison with the literatures and have to make sure that the values of Specificity and Sensitivity are near as much as possible to ensure their liability of the diagnosis system.

\section{Results And Discussion}

The results of this research are mostly numbers. The most important criteria of the results are the accuracy of classifying the images. In other words, the most important factor that is being examined is how accurate the classifier is in classifying the two brain conditions. For this paper's purpose, the accuracy and error are the most important parameters since they determine how good the classifier is. The TCIA datasets MRI Images were binary classified as Benign vsMalignant cases by features extractions were extracted thefeatures of the firstorder statistical asmentioned in feature extraction paragraph and the evaluationof the two classifiers (support vector machine $(\mathrm{SVM})$ and $\mathrm{K}$-voting Nearest Nieghbor $(\mathrm{KNN})$ ) were learning the system by $57 \%$ of datasets and 
Signal \& Image Processing: An International Journal (SIPIJ) Vol.11, No.1, February 2020

testing them by $43 \%$ all these processing has beencarried out in MATLAB R2019b software, furthermore the same selected features parameters are applying on each classifieras shown their results below inTable.1.The table presents the accuracy and error of the eight classifiers. Those parameters are important in determining the best classifier that should be used for such a software or application.

Table 2. Results of two classifiers

\begin{tabular}{|l|l|l|l|l|l|}
\hline Classifier & Sensitivity & Specificity & Accuracy & Error & AUC \\
\hline SVM "Linear" & 96.77 & 100 & 97.78 & 3.33 & 96.67 \\
\hline SVM "rbf" & 100 & 100 & 100 & 0 & 100 \\
\hline $\begin{array}{l}\text { SVM } \\
\text { "PolynomialOrder2" }\end{array}$ & 100 & 100 & 100 & 0 & 100 \\
\hline $\begin{array}{l}\text { SVM } \\
\text { "PolynomialOrder3" }\end{array}$ & 100 & 100 & 100 & 0 & 100 \\
\hline KNN K=1 & 100 & 100 & 100 & 0 & 100 \\
\hline KNN K=3 & 96.77 & 100 & 97.78 & 3.33 & 96.67 \\
\hline KNN K=5 & 93.75 & 100 & 95.56 & 6.67 & 93.33 \\
\hline
\end{tabular}

\section{CONCLUSION}

This research's aim is to design a software that automatically classifies brain images into twodifferent classes. The classes hereare normal and abnormal images. The special thing about this research is that the software will classify normal and abnormal brain images. For the purpose of this research, only one brain condition is considered, Glioblastoma. In this research, an open source of brain images was used to train and test the designed software. The images were then analyzed and features were extracted from them. In this study, first order statistical features were considered. In order to successfully complete the research, seven different classifiers were used to classify the images. This was done to make sure the most accurate classifier is used. The classifiers were an SVM classifier in addition to a KNN classifier. According to our results, both SVM and KNN classifiers appeared to be the most accurate with an accuracy of $100 \%$

It is recommended in the future to use more images, obtain more features which will for sure increase the accuracy of the classifier. It is also recommended to develop the software to consider different brain diseases such as Alzheimer's.

\section{REFERENCES}

[1] "Clark K, Vendt B, Smith K, Freymann J, Kirby J, Koppel P, Moore S, Phillips S, Maffitt D, Pringle M, Tarbox L, Prior F. The Cancer Imaging Archive (TCIA): Maintaining and Operating a Public Information Repository, Journal of Digital Imaging, Volume 26,".

[2] "SAINI, Pankaj Kr; SINGH, Mohinder. Brain tumor detection in medical imaging using MATLAB. International Research Journal of Engineering and Technology, 2015, 2.02: 191-196."

[3] "ABD-ELLAH, Mahmoud Khaled, et al. Design and implementation of a computer-aided diagnosis system for brain tumor classification. In: 2016 28th International Conference on Microelectronics (ICM). IEEE, 2016. p. 73-76."

[4] "RAMARAJU, P. V.; SHAIK, B. Brain Tumour classification, Detection and Segmentation Using Digital Image Processing and Probabilistic Neural Network Techniques. International Journal of Emerging Trends in Electrical and Electronics, 2014, 10.10: 15-20." 
Signal \& Image Processing: An International Journal (SIPIJ) Vol.11, No.1, February 2020

[5] "MOHSEN, Heba; EL-DAHSHAN, El-Sayed Ahmed; SALEM, Abdel-Badeeh M. A machine learning technique for MRI brain images. In: 2012 8th International Conference on Informatics and Systems (INFOS). IEEE, 2012. p. BIO-161-BIO-165."

[6] "R. J. Ramteke, and K. Monali, “Automatic Medical Image Classification and Abnormality Detection Using K-Nearest Neighbour.” International Journal of Advanced Computer Research, vol. 2, no. 4(6) (2012)".

[7] "S. Chaplot, L. Patnaik, and N. Jagannathan, "Classification of magnetic resonance brain images using wavelets as input to support vector machine and neural network," Biomedical Signal Processing and Control, vol. 1, no. 1, pp. 86-92, 2006."

[8] "Abdullah, N., Ngah, U., and Azlin Aziz, S. "Image Classification of Brain MRI Using Support Vector Machine.” School of Electrical and Electronics Engineering".

[9] "Hamiane, M. and Saeed, F. (2017). "SVM Classification of MRI Brain Images for ComputerAssisted Diagnosis." International Journal of Electrical and Computer Engineering (IJECE), 7(5), p.2555."

[10] "M.C. Hingane, and S. B. Matkar, "Classification of MRI Brain Image using SVM Classifier", International Journal of Science Technology \& Engineering, 1 (9), 2015."

[11] [Online]. Available: https://www.mathworks.com/help/vision/feature-detection-andextraction.html.

[12] [Online]. Available: https://www.mathworks.com/help/stats/classificationlearner-app.html.

[13] [Online]. Available: http://www.saedsayad.com/model_evaluation_c.htm.

\section{Authors}

Umar S. Alqasemi, Assistant professor of Biomedical Engineering at King AbdulAziz University, researcher on biomedical engineering application, having more than twenty published papers, more than fifteen papers review and auditor, interested on photoacoustic imaging, CAD imaging, signal processing and more, guider on real life of biomedical business sector at KAU.

Ammar M. Alzuhair, Received bachelor degree in Medical Electronics Engineering from Kuala Lumpur University, Malaysia. Currently MSc. Electrical and Biomedical Engineering in King Abul-Aziz University, KSA.

Abdullah S. Bama'bad, Received bachelor degree in Biomedical Engineering from King Abdul-Aziz University, KSA. Currently MSc. Electrical and Biomedical Engineering in King Abul-Aziz University, KSA.

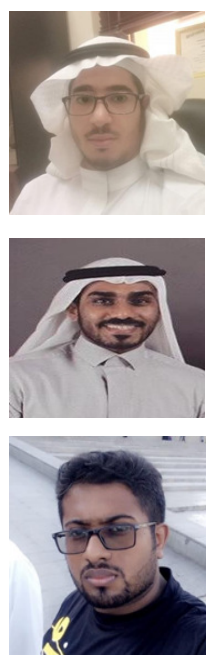

